Lance Cooley (FNAL), David

Larbalestier (NHMFL-FSU),

Arup Ghosh (BNL), Alvin

Tollestrup (FNAL)

Cross-cutting / Discovery

Science

\title{
Materials for accelerator technologies beyond the niobium family
}

Summary: Three niobium-based materials make up the entire present portfolio of superconducting technology for accelerators: $\mathrm{Nb}$ - $\mathrm{Ti}$ and $\mathrm{Nb}_{3} \mathrm{Sn}$ magnet wires and pure niobium for RF cavities. Because these materials are at a high level of maturity, limits imposed by the boundaries of their superconductivity constrain the energy reach of accelerators to several TeV. We sketch here a plan for targeted development of emerging higher field and higher temperature superconductors that could enable accelerators at significantly higher energies.

Present status: Niobium-based superconductors are the crucial enablers of present accelerators. The Nb-Ti LHC dipole and quadrupole wires, with transition temperature $\mathrm{T}_{\mathrm{c}}$ of $9 \mathrm{~K}$ and upper critical field $\mathrm{H}_{\mathrm{c} 2}$ of $15 \mathrm{~T}$, represent the highest form of superconductor strand art: massive, quarter-ton conductor billets are drawn from $300 \mathrm{~mm}$ diameter to $\sim 1 \mathrm{~mm}$ as a single, multi-kilometer-long piece, while retaining uniformity of the several thousand $\mathrm{Nb}$-Ti filaments to within $5 \%$ at the scale of a few micrometers. Strands are twisted into fully transposed cables with virtually no loss, preserving a carefully tuned nanostructure that generates the high fluxpinning forces and high current densities to enable high magnetic fields. $\mathrm{Nb}_{3} \mathrm{Sn}$, with twice the $\mathrm{T}_{\mathrm{c}}$ and $\mathrm{H}_{\mathrm{c} 2}$, is now approaching this level of conductor art, where over the last 5 years the LHC Accelerator Research Program (LARP) and the Next European Dipole (NED) program have demonstrated that $\mathrm{Nb}_{3} \mathrm{Sn}$ can be made into 4 meter long quadrupoles with $12 \mathrm{~T}$ fields and 250 $\mathrm{T} / \mathrm{m}$ gradients. Linear accelerators at TJNAF, ORNL (SNS), and under construction for the European XFEL exploit niobium superconducting radio-frequency (SRF) technology, with gradients at $\sim 20 \mathrm{MV} / \mathrm{m}$. Tremendous research and development is underway to realize highpower goals for Project X at FNAL and for a possible ILC at $35 \mathrm{MV} / \mathrm{m}$ gradients.

Despite these impressive achievements, the very maturity of these niobium-based technologies makes them incapable of additional leaps from the several-TeV scale. $\mathrm{Nb}-\mathrm{Ti}$ is already nearly perfect and operates at the limit of the superconducting phase. Further perfection of $\mathrm{Nb}$ cavities and $\mathrm{Nb}_{3} \mathrm{Sn}$ magnets might provide $50 \%$ growth in energy, based on proof-ofprinciple demonstrations that approach theoretical limits, e.g. $52 \mathrm{MV} / \mathrm{m}$ gradient in re-entrant $\mathrm{Nb}$ cavities and $18 \mathrm{~T}$ dipoles made from $\mathrm{Nb}_{3} \mathrm{Sn}$ strand. However, operation close to 
superconducting margins is risky, and cost tradeoffs to execute such a high degrees of perfection are likely to be negative.

Future possibilities: Fortunately there are emerging superconducting materials that have superconducting boundaries many times higher than those of $\mathrm{Nb}$-based materials. These offer the possibility for transformational changes in accelerator technology for discovery science while providing ample engineering headroom. Some, especially the 3 cuprate materials $\mathrm{Bi}_{2} \mathrm{Sr}_{2} \mathrm{CaCu}_{2} \mathrm{O}_{\mathrm{x}}(\mathrm{Bi}-2212),(\mathrm{Bi}, \mathrm{Pb})_{2} \mathrm{Sr}_{2} \mathrm{Ca}_{2} \mathrm{Cu}_{3} \mathrm{O}_{\mathrm{x}}(\mathrm{Bi}-2223)$ and $\mathrm{YBa}_{2} \mathrm{Cu}_{3} \mathrm{O}_{7-\delta}(\mathrm{YBCO})$, already exist in forms adaptable to accelerator technology. These so-called high temperature superconductors (HTS) have transition temperatures of $90 \mathrm{~K}$ or more, 5 times that of $\mathrm{Nb}_{3} \mathrm{Sn}$ and 10 times that of $\mathrm{Nb}$ and $\mathrm{Nb}-\mathrm{Ti}$, and $\mathrm{H}_{\mathrm{c} 2}$ of $\sim 120 \mathrm{~T}$ at $4 \mathrm{~K}, \sim 4$ times that of $\mathrm{Nb}_{3} \mathrm{Sn}$. Focused programs over 5 years in the areas described shortly could bridge gaps between such emerging materials with promising properties and the first magnet conductors or RF cavities with properties superior to those possible in $\mathrm{Nb}$-based materials.

One example of a route to this future is provided by the just started Very High Field Superconducting Magnet Collaboration (VHFSMC), whose goal is to develop the conductor and magnet technology of the Bi-2212 cuprate superconductor. The overall goal of VHFSMC is to demonstrate a viable conductor and coil winding technology using Bi-2212 conductors. Bi-2212 is the only HTS conductor that can develop high current densities in round wire form, which enables the manufacture of the Rutherford cables used for accelerator magnets. VHFSMC unites the materials science, the fabrication skills, the magnet construction expertise and the accelerator visionaries of 3 DOE-HEP labs, 3 universities, one other government lab, and US industry to interactively pull this new technology as far and as fast as possible. All key issues are being attacked: (a) understanding the fabrication and materials science of the superconductor in conductor forms, (b) understanding of superconductor leaks through the surrounding matrix and other unwanted interactions with insulation and structural materials, (c) assessment of the electromagnetic stability and magnet quench management, (d) understanding of the mechanical limitations of conductors, (e) development of cables and small coils, and (f) partnering with U.S. industry to ensure continued strand improvement and cost reduction. Such a consortium represents a general framework for how other targeted developments such as those noted below could be conducted.

Proposed efforts: Additional programs in the following areas could have high impact on long-term accelerator technology:

- The key obstacle to development of HTS conductors with flexible architectures similar to those in $\mathrm{Nb}$ conductors is the tendency of cuprate grain boundaries to have depressed superconducting properties. Although working models of simple, planar grain boundaries that consider modification of the superconducting properties due to strain and charge can explain many aspects of the role of grain boundaries, the "real" grain boundaries found in conductor-form materials are both more complex and often better. 
The highly desirable properties of Bi-2212 in round wire form are unique among all other HTS cuprate materials so far, and this fact may be explained by the overdoping that can occur in $\mathrm{Bi}-2212$ but not in $\mathrm{YBCO}$ and $\mathrm{Bi}-2223$. However, for fundamental reasons associated with its layered atomic structure, Bi-2212 can only be used to generate fields of $20 \mathrm{~T}$ or more at temperatures below $\sim 15 \mathrm{~K}$. A round wire form of YBCO could enable high-field operation as high as $55 \mathrm{~K}$ ! Little attention has been directed thus far to round wire YBCO because electricity applications above $50 \mathrm{~K}$ require imminent and cheap conductors that are reasonably well addressed by the presently available tape form. Such forms are not at present, however, suitable for accelerator applications. Targeted programs at understanding in detail the ways to enhance the properties of real grain boundaries could pay huge dividends in allowing the flexible conductor architectures, especially round wire forms, that magnet builders prefer.

- The route to a useful accelerator conductor using such tapes is not completely closed however. In pursuit of a multi-kiloamp, transposed cable, YBCO tapes of the type being produced now under the sponsorship of the DOE Office of Electricity Distribution and Energy are being made into Roebel cables. Development programs that incorporate such tape conductors into accelerator technologies could take advantage of the tremendously high upper critical field and nearly field-independent current density of YBCO over a wide range of temperature and field. As these conductors are now available industrially from two US companies, now is the time to explore alternatives to conventional Rutherford cabling, management of high integrated current with time (MIITs), and the understanding of the mechanical and performance limits of such materials.

- RF cavity performance is presently limited by a complex mix of fabrication compromises and uncertainties about the true properties of the $40 \mathrm{~nm}$ of interior surface over which RF supercurrents flow and by the desire to work with much higher $T_{c}$ and critical field $\mathrm{H}_{\mathrm{c}}$ materials. At present, $\mathrm{Nb} \mathrm{RF}$ cavities are processed using a subtractive paradigm, in which mechanical means and chemical etching are used to remove fabrication artifacts and prepare the final RF surface. The opposite paradigm of growing films of pure $\mathrm{Nb}$, $\mathrm{NbN}$, or $\mathrm{Nb}_{3} \mathrm{Sn}$ using physical or chemical vapor deposition, when allied with recent developments from nanomaterials synthesis, may provide the needed surface control at the level of individual monolayers, well below the $40 \mathrm{~nm}$ scale at which supercurrents flow in $\mathrm{Nb}$. Higher purity, better composition control, and smoothing of substrate defects are attractive features of these new deposition techniques. Moreover, a recent fundamental rethink of the physics of RF cavities suggests a new paradigm in which the final RF surface is built up from higher $\mathrm{T}_{\mathrm{c}}$ and higher $\mathrm{H}_{\mathrm{c}}$ materials interleaved with insulators. Hybrids that incorporate dielectrics and materials with tuned work functions would also be possible with such technologies. Theoretical understanding suggests that coatings capable of gradients above $100 \mathrm{MV} / \mathrm{m}$ are possible. 
Several consortia are already positioned to respond to a broad agency announcement covering accelerator technologies beyond niobium-based materials. An incomplete list appears below:

- The VHFSMC, which consists of FNAL, Florida State / NHMFL, BNL, LBNL, NIST, Texas A\&M, LANL, and shares close ties with Oxford Instruments - Superconducting Technology, Supercon Inc., and Superconductive Components Inc.

- The HTS wire development consortia, including ORNL, LANL, BNL, ANL, University of Houston, and Florida State / NHMFL with close ties to American Superconductor Corp. and Superpower, Inc.

- The Air Force Research Laboratory and the Naval Research Laboratory

- RF cavity consortia including TJNAF, Old Dominion University, College of Wm. \& Mary, Virginia Tech, Cornell, LBNL, ANL, Stanford, LANL, Fermilab, Temple University, University of Maryland, Michigan State, Florida State / NHMFL. 